BMC

Genomics

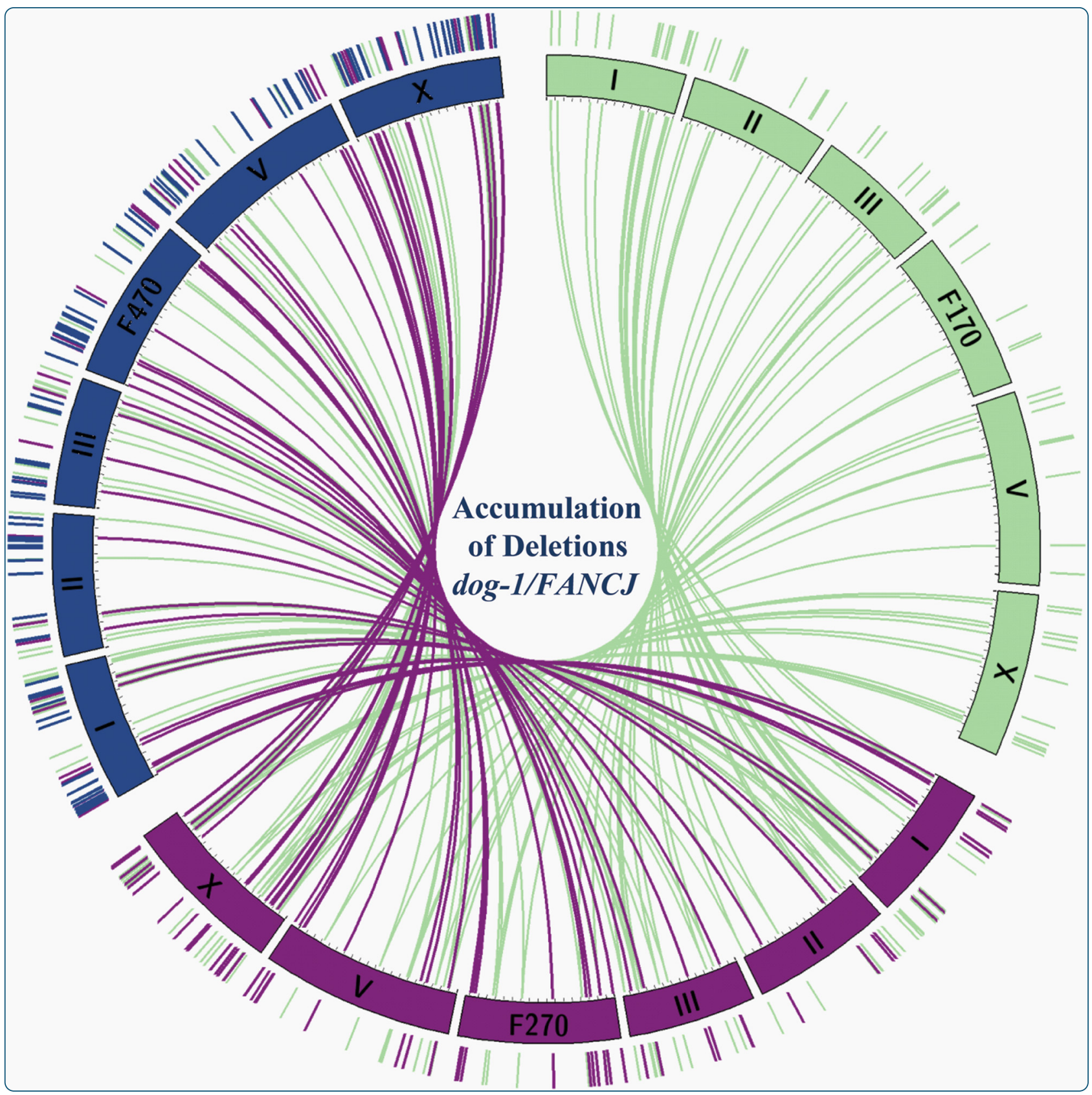

Spectrum of variations in dog-1/FANCJ and mdf-1/MAD1 defective Caenorhabditis elegans strains after long-term propagation

Tarailo-Graovac et al. 


\title{
Spectrum of variations in dog-1/FANCJ and mdf-1/MAD1 defective Caenorhabditis elegans strains after long-term propagation
}

Maja Tarailo-Graovac 1,3,4,5*, Tammy Wong ${ }^{1}$, Zhaozhao Qin' ${ }^{1}$ Stephane Flibotte², Jon Taylor ${ }^{2}$, Donald G Moerman², Ann $M$ Rose $^{3}$ and Nansheng Chen ${ }^{1 *}$

\begin{abstract}
Background: Whole and partial chromosome losses or gains and structural chromosome changes are hallmarks of human tumors. Guanine-rich DNA, which has a potential to form a G-quadruplex $\left(\mathrm{G}_{4}\right)$ structure, is particularly vulnerable to changes. In Caenorhabditis elegans, faithful transmission of G-rich DNA is ensured by the DOG-1/FANCJ deadbox helicase.

Results: To identify a spectrum of mutations, after long-term propagation, we combined whole genome sequencing (WGS) and oligonucleotide array Comparative Genomic Hybridization (oaCGH) analysis of a C. elegans strain that was propagated, in the absence of DOG-1 and MDF-1/MAD1, for a total of 470 generations, with samples taken for long term storage (by freezing) in generations 170 and 270. We compared the genomes of $F_{170}$ and $F_{470}$ strains and identified 94 substitutions, 17 InDels, 3 duplications, and 139 deletions larger than 20 bp. These homozygous variants were predicted to impact 101 protein-coding genes. Phenotypic analysis of this strain revealed remarkable fitness recovery indicating that mutations, which have accumulated in the strain, are not only tolerated but also cooperate to achieve long-term population survival in the absence of DOG-1 and MDF-1. Furthermore, deletions larger than 20 bp were the only variants that frequently occurred in G-rich DNA. We showed that 126 of the possible 954 predicted monoG/C tracts, larger than 14 bp, were deleted in unc-46 mdf-1 such-4; dog-1 $\mathrm{F}_{470}$ (JNC170).
\end{abstract}

Conclusions: Here, we identified variants that accumulated in C. elegans' genome after long-term propagation in the absence of DOG-1 and MDF-1. We showed that DNA sequences, with $\mathrm{G}_{4}$-forming potential, are vulnerable to deletion-formation in this genetic background.

Keywords: C. elegans, Whole genome sequencing (WGS), oligonucleotide array Comparative Genomic Hybridization (oaCGH), Mutation accumulation (MA), Genomic variation (GV), Spindle assembly checkpoint (SAC), dog-1/FANCJ,

G-quadruplex $\left(G_{4}\right)$ structure

\section{Background}

Genome integrity is crucial for survival of all living organisms. Chromosomal instability (CIN), marked by whole or segmental aneuploidy is hallmark of human tumors, and can drive abnormal proliferation of cancer cells [1]. In Caenorhabditis elegans, $m d f-1(g k 2)$ has an essential $m d f-1 /$ $M A D-1$ component of the spindle assembly checkpoint

\footnotetext{
*Correspondence: maja@cmmt.ubc.ca; chenn@sfu.ca

'Department of Molecular Biology and Biochemistry, Simon Fraser University, V5A 156 Burnaby, BC, Canada

${ }^{3}$ Department of Medical Genetics, University of British Columbia, V6T 1 Z3 Vancouver, BC, Canada

Full list of author information is available at the end of the article
}

(SAC) missing and this leads to accumulation of genetic errors and ultimately death by the third generation [2]. The checkpoint prevents CIN by inhibiting anaphasepromoting complex/cyclosome (APC/C), and delaying anaphase onset until all the chromosomes have achieved proper attachment to the spindle [3].

While MDF-1 prevents both loss and gain of whole chromosomes during mitosis, DOG-1 prevents segmental aneuploidies by ensuring proper replication of guanine(G)-rich DNA [4-6]. G-rich DNA can adopt a four-stranded helical G-quadruplex $\left(\mathrm{G}_{4}\right)$ DNA structure [7-9], which can pose a barrier to replication fork 
progression if left unresolved. The ability to form $\mathrm{G}_{4}$ structures makes the corresponding G-rich DNA sequences particularly vulnerable to chromosomal rearrangements. Studies using C. elegans, as a model organism, were the first to show the striking genomic instability of G-rich DNA sequences when DOG-1, a functional ortholog of the deadbox helicase FANCJ [10], was non-functional [4]. When DOG-1 is functional, G-rich DNA sequences are stable and deletions affecting these regions are not observed [4,5]. Genomewide bioinformatic analysis of the human genome had identified more than 300,000 DNA sites with $\mathrm{G}_{4}$-forming potential $[11,12]$. In humans, mutations in FANCJ/dog-1 have been identified in Fanconi anemia (FA) complementation group J patients [13-15], which is a severe, autosomal recessive, disorder with increased spontaneous and DNA crosslink-induced CIN showing a wide range of clinical manifestations [13], and also in early onset breast cancer patients $[16,17]$.

Knowledge of the mutational spectra is crucial for deciphering the cause of heritable genetic disorders as well as the progression of events relevant to cancers. Traditionally, analyses of mutation spectra and rates have been based on a small portion of phenotypically and molecularly detectable loci. In C. elegans, the mutational spectrum of $d o g-1(g k 10)$ (knockout allele of $d o g$ 1) strains, was analyzed using either PCR-based assays $[4,10,18]$ or oligonucleotide array Comparative Genomic Hybridization (oaCGH) $[5,6,19]$. The rapid advances in "next-generation" DNA sequencing technologies now allows us to perform comprehensive genome-wide analyses of mutational spectra by sequencing whole genomes [20-23].

Here we undertook a whole genome approach in order to analyze mutational events in a $C$. elegans strain that is defective for both $m d f-1 / M A D 1$ and $\operatorname{dog}-1 /$ FANCJ. This strain was propagated for 470 generations and samples were stored frozen at generations 170 and 270. Phenotypic analysis of the strain revealed striking fitness recovery, indicating that accumulated mutations cooperate to bypass the MDF-1 checkpoint requirement and thus achieve long-term population survival. We performed whole-genome sequencing (WGS) and oaCGH analyses of the strain at three different generations (170, 270 and 470). We identified substitutions, InDels, and copy number variants (CNVs) larger than $20 \mathrm{bp}$, and compared their accumulation over the generations. We showed that only deletions, which are larger than $20 \mathrm{bp}$, frequently initiated in G-rich DNA ( $88 \%$ of all of the deletions). Consistent with the fitness recovery observed in this strain, rather than a decline in fitness, the mutation spectrum reported here reflects variants that are either advantageous or neutral in this specific genetic background.

\section{Results and discussion}

\section{Whole genome analysis}

In order to propagate $m d f-1$; $d o g-1$ homozygous worms, it is first necessary to isolate a suppressor of $m d f-1(g k 2)$ sterility and lethality that occurs in the double mutant [24]. Previously, such-4(h2168) was isolated, which allowed propagation of $m d f-1$; $d o g-1$ beyond the third generation (Figure 1) [24]. The such-4 suppressor allows for an approximately five-fold increase in fertile hermaphrodite progeny of $m d f-1 ; \operatorname{dog}-1$ [24]. This increase in fertility occurs in the generations immediately after isolation of the suppressor. We outcrossed one worm to obtain KR4233 [unc-46(e177) mdf-1(gk2) such-4(h2168)] [24,25] (Figure 1). A second line was isolated and maintained in the $\operatorname{dog}-1$ ( $g k 10)$ background for 470 generations, with storage by freezing at generations 170 and 270 (Figure 1). To mark the presence of $m d f-1(g k 2)$, unc46(e177) (a visible marker) was used, which is present in all of our strains (Figure 1). While propagating unc-46 mdf-1 such-4; $\operatorname{dog}-1$ homozygotes, we observed a further increase in reproductive fitness. This increase was significant, $59 \%$ of $\mathrm{F}_{470}$ unc-46 mdf-1 such-4; dog-1 progeny develop into fertile hermaphrodites, compared to only $10 \%$ of the unc- 46 mdf- 1 such-4 progeny and $2 \%$ of unc$46 m d f-1$ mutants. Detailed phenotypic analysis of these strains as well as genetic dissection of suppressors has recently been reported [26]. To identify the genomic variations (GVs) that had accumulated in mdf-1 such-4; $\operatorname{dog}-1 F_{470}$ worms after long-term propagation, the genome was sequenced to a depth of $70 \times$ genome equivalents and aligned to the $C$. elegans reference genome WS235 available at WormBase [27]. We also sequenced the unc-46 mdf-1 such-4; $\operatorname{dog}-1$ strains that were frozen at generations 170 and 270 and compared the progress of mutation accumulation (Additional file 1: Figure S1).

\section{Single Base Substitutions (SBSs) do not occur within $\mathrm{G}_{4}$-DNA}

We used the variant caller VarScan2; version 2.3.2 [28] to identify 525 homozygous SBSs that occurred with a variant frequency (VF) of $90 \%$ or higher in the $m d f-1$ such-4; $\operatorname{dog}-1_{\mathrm{F} 470}$ genome (Additional file 2: Table S1A). All 42 tested substitutions were confirmed by Sanger resequencing (30 were randomly selected; 12 additional substitutions were confirmed by re-sequencing as a result of being adjacent to randomly selected SBSs or as being non-randomly selected as candidates in later analyses), indicating a false positive rate of less than $5 \%$. WGS analysis of the unc-46 mdf-1 such-4; dog-1 strain, which had been frozen at generation 170 (Figure 1), revealed that the majority (431/525) of the SBSs present in generation 470 are also present in generation 170 (Additional file 1: Figure S1A; Table 1). However, we did observe that 32 additional substitutions had accumulated between $\mathrm{F}_{170}$ 


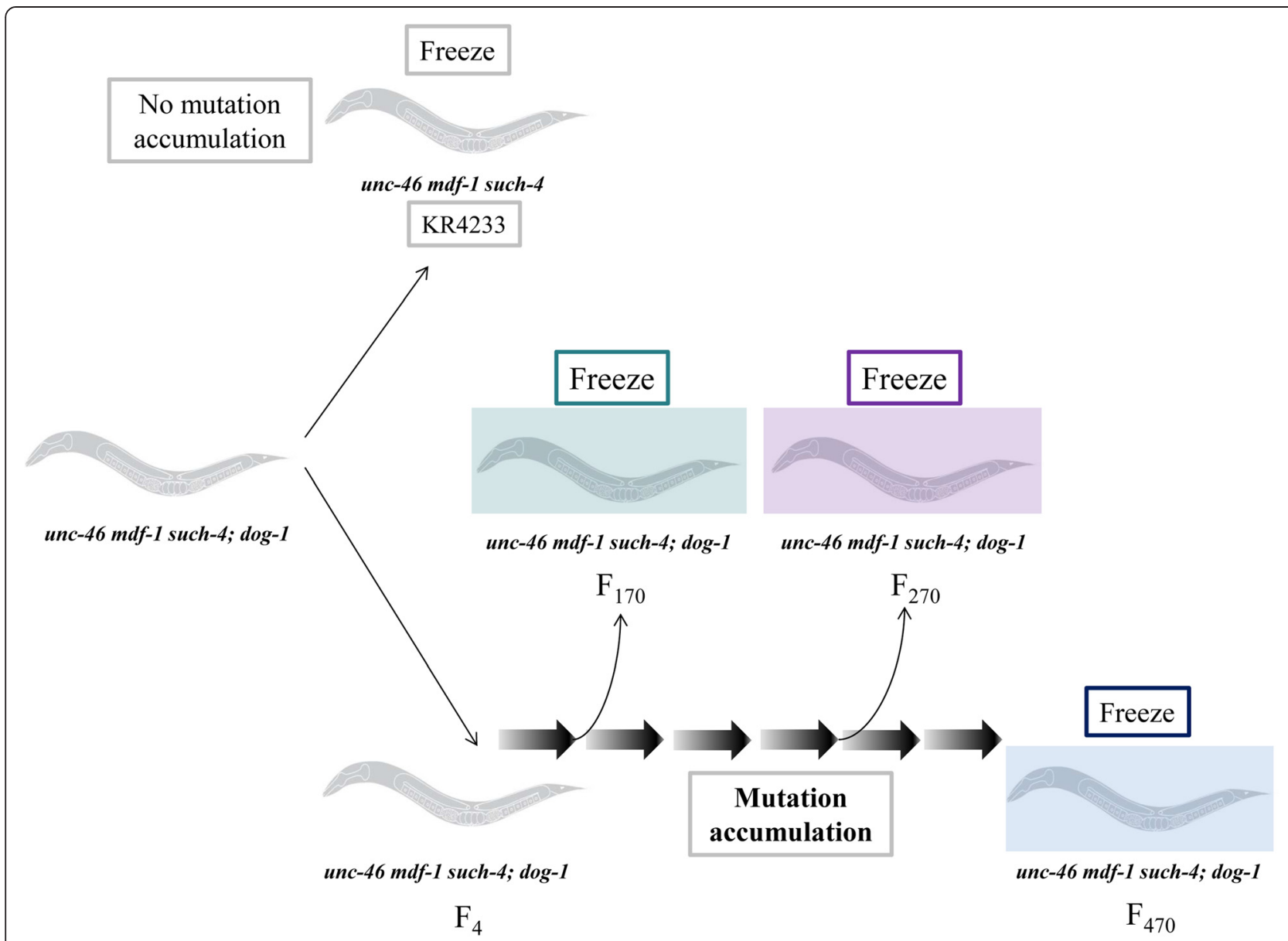

Figure 1 A schematic representation of the long-term propagation of unc-46 mdf-1 such-4; dog-1 homozygotes for 470 generations. We isolated a suppressor (such-4) in $\mathrm{F}_{4}$ from the only plate that we set up that propagated in the absence of MDF-1 and DOG-1 for longer than three generations. We generated a strain (KR4233) by crossing away dog-1(gk10). A second line was propagated for the total of 470 generations. The worms were frozen at generations 170 (green), 270 (purple) and 470 (blue). Note that unc-46 (used as a visible marker) is tightly linked to $m d f-1(g k 2)$ in all of our strains.

and $\mathrm{F}_{270}$, and 62 more between $\mathrm{F}_{270}$ and $\mathrm{F}_{470}$ (Table 1). Large number of substitutions observed in $\mathrm{F}_{170}$ indicate the possibility that the original unc-46 mdf-1 such-4; dog-1 strain (Figure 1) had a large number of single nucleotide differences from the reference genome (WS235). One way to test this possibility is to estimate the mutation rates based on available data. If we consider the 94 substitutions that had accumulated in 300 generations, between $F_{170}$ and $\mathrm{F}_{470}$, we estimate the rate of $\mu_{\mathrm{bs}}=3.1 \times 10^{-9} / \mathrm{base} /$ generation. This estimate is similar to the previously reported spontaneous rate of base substitution in C. elegans, $2.7 \pm 0.4 \times 10^{-9} /$ base/generation [21] and other model organisms, $3.5 \times 10^{-9} /$ base/generation in Drosophila melanogaster [23] and $7.1 \pm 0.7 \times 10^{-9} /$ base/generation in Arabidopsis thaliana [22]. Furthermore, analysis of the 94 substitutions on mutation bias revealed very similar mutation spectrum to the spontaneous mutation spectra in N2 (C. elegans wild-type) [21] (Figure 2), and our analysis of transition bias (transition/transversion - Ts/Tv base substitution ratios) revealed the $\mathrm{Ts} / \mathrm{Tv}$ ratio $=0.54$,

Table 1 Summary of the variants identified in the unc-46 mdf-1 such-4; dog-1 strains at generations $F_{170}, F_{270}$ and $F_{470}$

\begin{tabular}{lllll}
\hline & SBSs & InDels $\leq \mathbf{2 0}$ bp & Duplications & Deletions $>\mathbf{2 0}$ bp \\
\hline F170 & 431 & 133 & 3 & 57 \\
F270 & 32 & 8 & $3^{*}$ & 45 \\
F470 & 62 & 9 & -1 & 94 \\
Total F170-F470 & 94 & 17 & 3 & 139
\end{tabular}

*Two new duplications and amplification of the cyb-3 locus to three copies. 


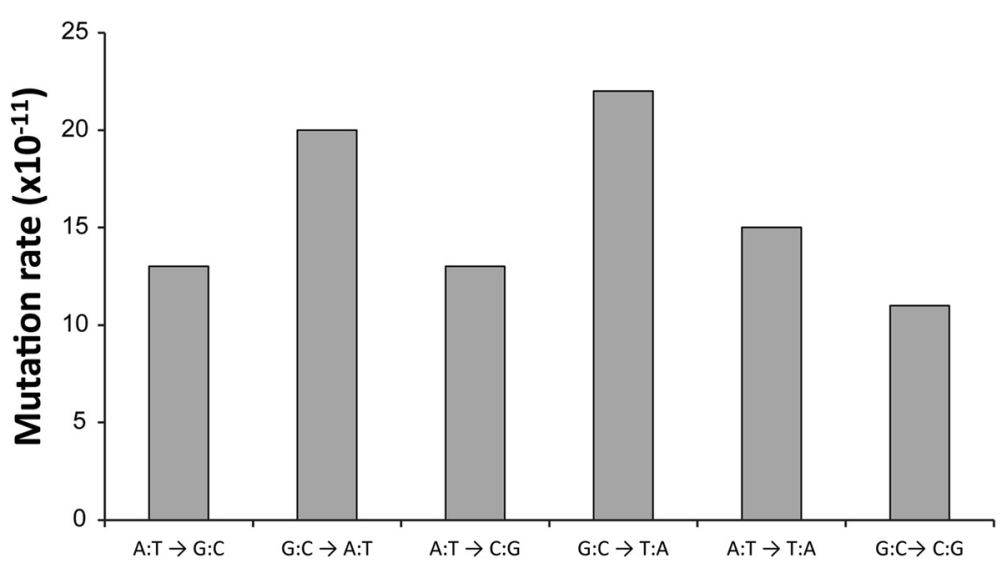

Figure 2 Mutation rate estimates. The variants analyzed are the 94 SBSs that were identified between generations 170 and 470 .

which is within the range observed for spontaneous mutations in multiple mutation accumulation lines (average 0.45 ; range $0.19-0.79$ ) [21]. Therefore, the similarity of the substitution rate over the last 300 generations to the previously reported $\mu_{\mathrm{bs}}$ in N2 strongly implies that the majority of the 431 substitutions identified in the $F_{170}$ generation are variants originally present in the starting strain; therefore, we focused our analysis on GVs that had occurred between $F_{170}$ and $F_{470}$ (Additional file 2: Table S1B).

Previous studies showed that $\mathrm{G}_{4}$ DNA secondary structure is mutagenic in the absence of DOG-1 [4-6,19]. Using the $\mathrm{G}_{4}$ DNA signature $\left(\mathrm{G}_{3+} \mathrm{N}_{1-7} \mathrm{G}_{3+} \mathrm{N}_{1-7} \mathrm{G}_{3+} \mathrm{N}_{1-7} \mathrm{G}_{3+}\right)$, we identified 2,372 such sites in C. elegans' genome (Additional file 2: Table S2). Next, we tested to see if any of the 94 substitutions occurred within the $\mathrm{G}_{4}$ DNA signature sequence and observed that none of them did (Additional file 2: Table S1B), indicating that the identified substitutions most likely arose spontaneously and were not due to lack of functional DOG-1.

\section{Small InDels do not occur within $\mathrm{G}_{4}$-DNA}

We applied VarScan2 [28] to identify 150 homozygous InDels that were 20 bp or less in mdf-1 such-4; $d o g-1_{\text {F470 }}$ (Additional file 2: Table S3A). We randomly selected 25 of the InDels and confirmed all 25 by Sanger re-sequencing, indicating a false positive rate of less than $5 \%$ (Additional file 2: Table S3A). Analysis of InDels in the unc-46 mdf-1 such-4; dog-1 strains propagated for 170 and 270 generations (Additional file 1: Figure S1B) revealed that $88 \%$ of the $F_{470}$ InDels (133/150) existed in the $F_{170}$ generation (Table 1), which again indicates that the majority of InDels were already present in our starting strain. In fact, we only observe eight additional InDels in $F_{270}$ (Table 1) and another nine InDels accumulated between $F_{270}$ and $F_{470}$ (Table 1). Based on the last 300 generations of propagation, we estimate a mutation rate for InDels to be $17 /(1 \times$ $\left.10_{\text {bases }}^{8} \times 300_{\text {generations }}\right)=5.7 \times 10^{-10} /$ base/generation. The ratio of InDels to SBSs observed was 0.18 or one InDel per 5.5 substitutions, which is much lower than the 1.31 ratio reported previously in C. elegans [29]. However, the lower number of InDels to SBSs that we observe in the unc-46 mdf-1 such-4; dog-1 background is comparable to analyses in yeast [30], A. thaliana [22] and human [31]. Namely, WGS analysis in yeast had revealed ratio of InDels to SBSs of $\sim 0.03$ [30], which was consistent with previous findings of one InDel per 33 SBSs [20]. Furthermore, analysis on spontaneous occurrence of InDels in $A$. thaliana revealed the ratio of 0.13 of InDels to SBSs [22]. It may be possible that the small number of InDels, over the last 300 generations, in our strain may be a result of a mutation that was acquired by propagation; however, it may also be that the spontaneous mutation rate of InDels in C. elegans is comparable to that of other organisms.

Next, we tested to see if any of the 17 InDels occurred within the $G_{4}$ DNA signature sequence and observed that none of them did (Additional file 2: Table S3B). Thus, we believe that these InDels arose spontaneously and are not due to a lack of functional DOG-1.

\section{Duplications do not occur within $\mathrm{G}_{4}$-DNA}

Analysis of gene copy-number variant accumulation after long-term propagation in C. elegans using the oaCGH has provided evidence for a high rate of spontaneous gene duplications in this multi-cellular eukaryote [32]. Previously, using the oaCGH we showed that the such-4 suppressor genome contains a large tandem duplication on Chromosome V (LGV) [6]. Here, we used both Pindel [33] and oaCGH [34], and identified four sites with copy number increases in the $m d f-1$ such-4; $d o g-1_{\mathrm{F} 470}$ genome (Additional file 2: Table S4A), including a previously identified large tandem duplication [6]. One of the duplications involves a two-copy addition, making the final count of five duplication events and four different duplication sites. Analysis of the CNVs in the $\mathrm{F}_{170}$ and $\mathrm{F}_{270}$ genomes captured a dynamic property of duplications (Additional 
file 1: Figure $\mathrm{S} 1 \mathrm{C})$. In $\mathrm{F}_{170}$, we observed three duplications (Additional file 2: Table S4A). One is the large tandem duplication located on LGV that amplifies 62 protein-coding genes, which we have described previously [6]. In generation $\mathrm{F}_{270}$, we detected duplications of two new loci, as well as further amplification of the LGV region to three copies (triplication) (Additional file 1: Figure S1C and Additional file 2: Table S4A). In $\mathrm{F}_{470}$, we did not find any new duplications (Additional file 2: Table S4A), but did observe that the duplication on LGI was lost, resulting in a wild-type copy number for this region (Additional file 2: Table S4A). Thus, the LGV duplication exemplifies the property of duplications to further amplify; while LGI duplication shows that a duplicated region can revert back to a normal copy number.

The gene duplication rate for C. elegans was recently estimated to be $3.4 \times 10^{-7} /$ gene/generation [32]. Our data, based on the last 300 generations (from $F_{170}$ and $F_{470}$ ), also revealed a comparably high rate of gene duplication $(6.5 \times$ $10^{-7}$ /gene/generation), specific to the unc-46 mdf-1 such-4; dog-1 background, when a large duplication on LGV is excluded from the analysis. The LGV duplicated region contains 62 protein-coding genes, including cyb-3 [6]. We observed a correlation between increased dosage of CYB-3 and a striking fitness increase in our strains; thus, our experimental protocol selected for LGV amplification (detailed experimental analysis on these findings has been recently published [26]). Importantly, like SBSs and InDels, none of the duplications occurred in the vicinity of the $G_{4}$ DNA signature sequences (Additional file 2: Table S4B), also indicating that these $\mathrm{CNVs}$ arose spontaneously and were not due to a lack of functional DOG-1.

\section{Deletions frequently initiate at $\mathrm{G}_{4}$ DNA sites}

The major type of mutation observed, in the absence of DOG-1, is a deletion of $300 \mathrm{bp}$ or smaller, initiating at either the 5 '-end of $\mathrm{C}$ - or the 3 '-end of G-tracts $[4,5,10,19]$. Using the unique alignments generated by Novoalign and Pindel [33], we identified 183 homozygous deletions larger than 20 bp in the $m d f-1$ such-4; $d o g-1_{F 470}$ genome (Additional file 2: Table S5A), including the known $m d f-1(g k 2)$ and $d o g-1$ ( $g$ k10) deletions. We randomly selected 28 of the deletions, and confirmed all of them using PCR (Additional file 2: Table S5A). To identify deletions, which may have been missed, we also used oaCGH [34]. We confirmed all of the deletions, predicted by Pindel, that were covered by oaCGH probes and identified an additional 13 deletions not detected by Pindel (Additional file 2: Table S5A), making the final count 196 homozygous deletions in the mdf-1 such-4; dog$1_{F 470}$ genome (Additional file 2: Table S5A).

We observed 57 deletions in $F_{170}$, indicating that 139 deletions had accumulated in 300 generations (between $F_{170}$ and $\mathrm{F}_{470}$ ) propagated in the absence of MDF-1 and DOG-1 (Additional file 1: Figure S1D and Table 1). We found that the majority of the deletions (123 of 139) initiated in $G_{4}$ DNA (Additional file 2: Table S5A). Previous analysis in $C$. elegans [32] estimated the spontaneous rate of deletions to be $2.2 \times 10^{-7} /$ gene/generation respectively. The 139 deletions accumulating between the generations $F_{170}$ and $F_{470}$ affect 19 protein-coding genes which allowed us to calculate the mutation rate of deletions over the 300 generations to be $19 /\left(20,400_{\text {protein-coding genes }} \times 300_{\text {generations }}\right)=3.1 \times 10^{-}$ 6/gene/generation in the unc-46 mdf-1 such-4; dog-1 strain, which is approximately 10-fold higher than the estimated rate in N2 [32]. This is similar to the estimated forward mutation frequency of $e T 1$-balanced lethal mutations in dog-1 ( $g k 10)$ background [6]. To determine if the elevated mutation rate of deletions is due to DOG-1 deficiency, we compared mutation rates in non- $\mathrm{G}_{4}$ sites versus mutation rate in the $\mathrm{G}_{4}$ sites. While the mutation rate of deletions affecting the non- $\mathrm{G}_{4}$ sites, $4.9 \times 10^{-7} /$ gene/generation or 5.0 $\times 10^{-10} /$ base/generation, is comparable to the previously reported spontaneous rate of deletions [32]; the mutation rate based on $\mathrm{G}_{4}$ sites, $1.7 \times 10^{-4} / \mathbf{G}_{\mathbf{4}}$ site/generation, illustrates the striking vulnerability of these DNA regions when DOG-1 is absent. Therefore, deletions larger than 20 bp are the only variants in the unc-46 mdf-1 such-4; $\operatorname{dog}-1$ strain that frequently occurred in the $\mathrm{G}_{4}$-DNA sites and had significantly higher mutation rate than the spontaneous rate reported previously for the strains with normal DOG-1 function.

\section{monoG/C tracts larger than $14 \mathrm{bp}$ are frequently deleted when DOG-1 is not functional}

In recent years, the $\mathrm{G}_{4}$ DNA has been implicated in diverse biological processes, such as gene expression [35] and DNA replication initiation [35]. Consistent with the established role of DOG-1, we found that the majority (114) of the homozygous deletions that we identified between $\mathrm{F}_{170}$ and $\mathrm{F}_{470}$ (139) initiated in the previously proposed $\mathrm{G}_{4}$ DNA signature $\mathrm{G}_{3+} \mathrm{N}_{1-7} \mathrm{G}_{3+} \mathrm{N}_{1-7} \mathrm{G}_{3+} \mathrm{N}_{1-7} \mathrm{G}_{3+}$ [5] (Additional file 2: Table S5B) where the G-tract was almost completely removed together with $5^{\prime}$ flanking DNA sequence (Figure $3 \mathrm{~A}$ and $\mathrm{B}$ ). In agreement with a previous study [5], we found that the majority of deletions initiate at monoG tracts larger than 14 bp (93 deletions) (Figure 3A), while 11 deletions initiate at monoG-like structures with no more than two nucleotides that interrupt the homopolymer (Figure 3B), and 10 deletions initiate at sequences that interrupt the homopolymers by three or more nucleotides (e.g., GGGtGGGGaagttatGG GaGGG) (Additional file 2: Table S5B). MonoG/C tracts larger than $14 \mathrm{bp}$ have the highest potential of forming the G-quartet structure. In fact, we find here that the unc-46 mdf-1 such-4; $\operatorname{dog}-1 \quad F_{470}$ genome has $13.2 \%$ of all the predicted monoG/C tracts larger than 14 bp deleted. An interesting question to be addressed with future research would be to determine how mutation rate changes with 


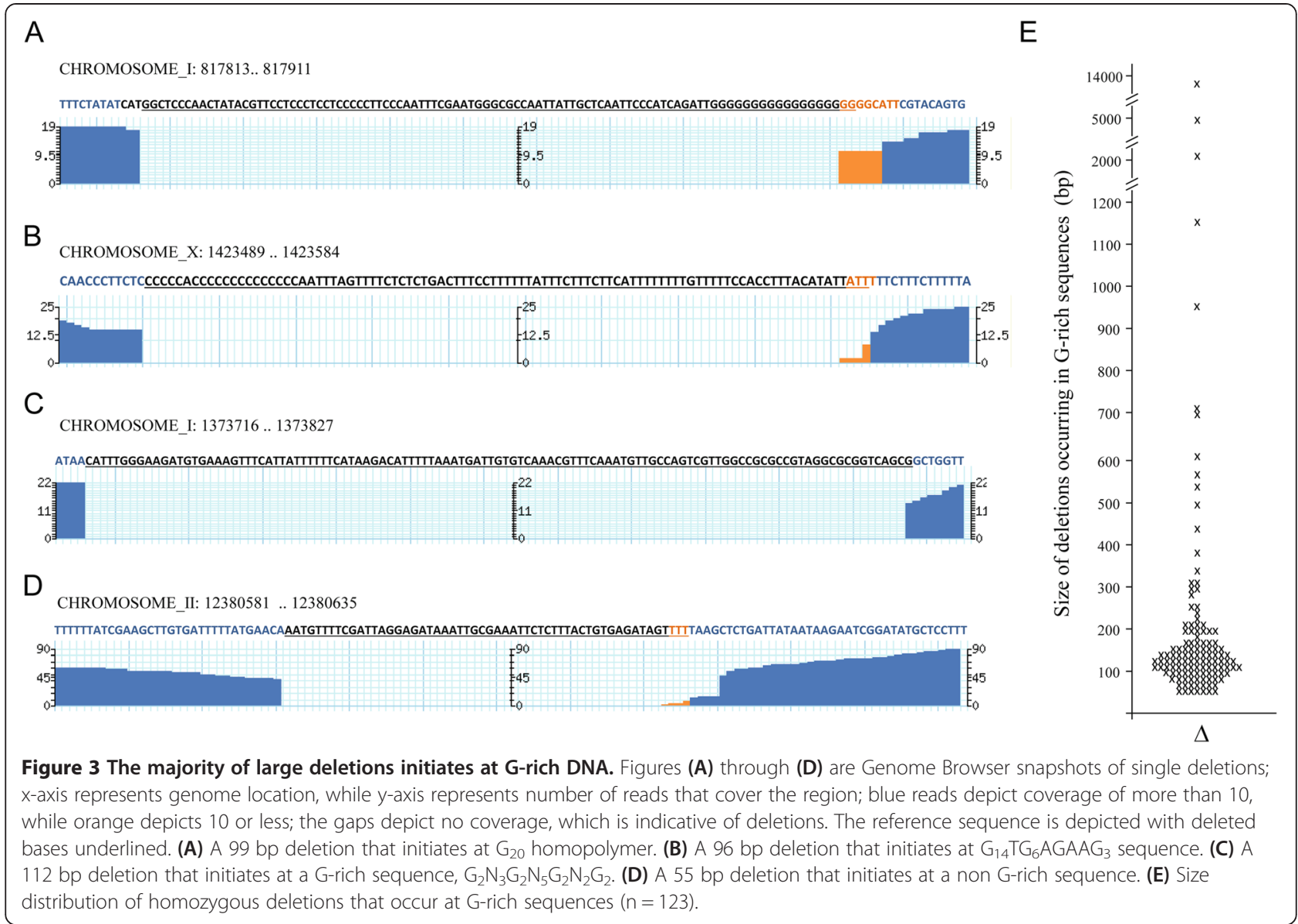

decreasing numbers of available targets. Furthermore, it would be also important to see how many of the $\mathrm{G}_{4}$-DNA sites could be deleted in a strain and still maintain viability of the animals.

To investigate whether there may be additional sequences, which are vulnerable in the absence of DOG-1, we analyzed the 25 deletions that do not initiate in $G_{4}$ DNA signature sequences to see if there are common patterns. We found that eight of these deletions initiate at $\mathrm{G}$-rich sequences that correspond to the $\mathrm{G}_{2+} \mathrm{N}_{1-7} \mathrm{G}_{2}$ ${ }_{+} \mathrm{N}_{1-7} \mathrm{G}_{2+} \mathrm{N}_{1-7} \mathrm{G}_{2+}$ signature (four stretches of two or more guanines, alternated with one to seven nucleotides of any type), while one had a $\mathrm{G}_{2+} \mathrm{N}_{1-7} \mathrm{G}_{2+} \mathrm{N}_{1-7} \mathrm{G}_{2+}$ signature at the breakpoint (Figure $3 \mathrm{C}$ and Table 2). Although, it may be possible that our strain had gained a mutation in an unknown gene important for genome stability, it is also possible that additional DNA sequences may be vulnerable to rearrangements in the absence of DOG-1.

Previously, it was found that the deletion sizes in viable lines detected by PCR $[4,18]$ or oaCGH methods $[5,6]$ were predominately smaller than $300 \mathrm{bp}$. In this study, the 123 deletions that occurred at G-rich sequences ranged between 49 and 10,228 base-pairs with the majority of the deletions (86\%) removing less than 300 bp (Figure 3E). These findings are in agreement with the deletion distribution sizes revealed by the study of a 69 G-tract deletion set [5]. However, larger deletions, initiating at G-tracts,

Table 2 Schematic representation of the nine deletions that initiate at G-rich regions within sequences that deviate from the $\mathbf{G} 4$ DNA signature, $\mathbf{G}_{3+} \mathbf{N}_{1-7} \mathbf{G}_{3+} \mathbf{N}_{1-7} \mathbf{G}_{3+} \mathbf{N}_{1-7} \mathbf{G}_{3+}$

\begin{tabular}{lll}
\hline LG & Locus & Signature \\
\hline I & $464950 . .465071$ & $\mathrm{G}_{2} \mathrm{NG}_{12}$ \\
$\mid$ & $815471 . .815611$ & $\mathrm{G}_{3} \mathrm{NG}_{3} \mathrm{NG}_{3} \mathrm{~N}_{2} \mathrm{G}_{2}$ \\
\hline & $3747905 . .3748027$ & $\mathrm{G}_{2} \mathrm{NG}_{2} \mathrm{NG}_{2} \mathrm{NG}_{2} \mathrm{NG}_{2} \mathrm{NG}_{2} \mathrm{NG}_{2} \mathrm{NG}_{2} \mathrm{NG}_{2}$ \\
\hline & 1373716.1373827 & $\mathrm{G}_{2} \mathrm{~N}_{3} \mathrm{G}_{2} \mathrm{~N}_{5} \mathrm{G}_{2} \mathrm{~N}_{2} \mathrm{G}_{2}$ \\
II & $12687460 . .12687546$ & $\mathrm{G}_{2} \mathrm{NG}_{14} \mathrm{NG}_{2}$ \\
III & $460436 . .460942$ & $\mathrm{G}_{11}$ \\
IV & $3298588 . .3298711$ & $\mathrm{G}_{14}$ \\
$V$ & $17778167 . .17778449$ & $\mathrm{G}_{3} \mathrm{NG}_{3} \mathrm{NG}_{3} \mathrm{~N}_{5} \mathrm{G}_{2} \mathrm{NG}_{2} \mathrm{NG}_{3}$ \\
$X$ & $2802061 . .28022233^{*}$ & $\mathrm{G}_{2} \mathrm{NG}_{3} \mathrm{~N}_{6} \mathrm{G}_{3}$ \\
\hline
\end{tabular}

*Analysis of the sequences revealed that nine match the $\mathrm{G}_{2+} \mathrm{N}_{1-7} \mathrm{G}_{2+} \mathrm{N}_{1-7} \mathrm{G}_{2+} \mathrm{N}_{1-}$ ${ }_{7} \mathrm{G}_{2+}$ signature, while one (marked with ${ }^{*}$ ) matches $\mathrm{G}_{2+} \mathrm{N}_{1-7} \mathrm{G}_{2+} \mathrm{N}_{1-7} \mathrm{G}_{2+}$ signature at the breakpoint. 
have been recovered as lethal mutations [6], consistent with these regions containing essential genes [6]. We also found that 16 deletions, which occurred at non-G-rich sites, removed small regions of less than $300 \mathrm{bp}$ in size (Additional file 2: Table S5B).

\section{1 protein-coding genes are affected by the 253 GVs}

To determine an effect of all the identified variants on protein-coding genes, after long-term propagation in the absence of MDF-1 and DOG-1, we used CooVar [36], a tool developed by our group for annotating variants. Many of the G-rich DNA sites are located in close proximity to protein-coding genes in C. elegans [19,37]. Using CooVar [36], we predicted that 19 genes would be affected by 18 of the deletions that had accumulated between generations $\mathrm{F}_{170}$ and $\mathrm{F}_{470}$ (Additional file 2: Table S6). The majority of the deletions (14) represent the first knockout alleles for those genes and thus provide a genetic resource for studying their functions (Additional file 2: Table S6). We found that the majority of the InDels are not located in proteincoding regions, and the one that affects a protein-coding gene is an in-frame deletion (Additional file 2: Table S6). We found that the majority of the detected SBSs are located within non-protein coding regions. However, there are 15 SBSs that fall within protein-coding regions that are predicted to result in missense mutations (Additional file 2: Table S6). In total, 66 protein-coding genes were affected by the duplications (Additional file 2: Table S6). The majority of the genes (62) are affected by the large tandem duplication on LGV (Additional file 2: Table S6). In addition to the $253 \mathrm{GVs}$ that had accumulated between generations $F_{170}$ and $F_{470}$, we had also performed analysis of the effect on protein-coding genes of all the variants observed in the mdf-1such-4; $\operatorname{dog}$ - $_{F 470}$ genome (Additional file 2: Table S7).

Mutations are a source of genetic variation that confers to an organism either advantage due to a beneficial change, disadvantage due to a deleterious alteration, or neither due to a neutral change. Considering the $\sim 30$-fold fitness recovery in this strain [26], the identified GVs are expected to be either advantageous or neutral in this genetic background. In fact we showed, in a parallel study, that three mutational events discovered in this strain cooperate to increase fitness when MDF-1 absent [26].

\section{Conclusion}

In this study we undertook a genomics approach to identify variants that accumulate in the $C$. elegans genome after long-term propagation in the absence of two genome-guardians, DOG-1/FANCJ and MDF-1/MAD1. Combining WGS analysis with oaCGH analysis allowed us to comprehensively analyze both the small-scale variants (SBSs and InDels of 20 bp or smaller) and largescale variants (CNVs larger than $20 \mathrm{bp}$ ). Freezing the strain, for long term storage, at three different time points, $F_{170}, F_{270}$ and $F_{470}$, allowed us to compare and visualize mutation accumulation over the generations. We were able to estimate the mutation rates in this strain for different types of mutations over the 300 generations $\left(F_{170}\right.$ to $\left.F_{470}\right)$. We observed a significantly elevated rate of deletions, larger than $20 \mathrm{bp}$, that initiated at G/C-rich DNA sequence. Our approach had allowed us to show that, in the absence of DOG-1, DNA sequences with $\mathrm{G}_{4}$-forming potential are vulnerable to deletion-formations. Previous studies were dependent only on PCR or oaCGH analysis; the two approaches are limited in their ability to detect small-scale variants like SBSs and InDels. Furthermore, genome analysis of the strains at $F_{170}, F_{270}$ and $F_{470}$ allowed us to capture and visualize an intriguing property of CNVs (Additional file 1: Figure S1). We did not find any reversions of deletions, substitutions, or InDels. As expected, once fixed, these types of mutations are propagated indefinitely (Additional file 1: Figure S1). However, we captured a dynamic property of duplications: amplification of a region on LGV from one-to-two-to-three copies and reversion of the LGI duplication back to a normal copy number (Additional file 1: Figure S1C).

This is the first extensive analysis of a strain that had been propagated in the absence of DOG-1 helicase for hundreds of generations. We identified 954 monoG/C tracts larger than $14 \mathrm{bp}$ in the $C$. elegans genome WS325; the polyG/C DNA sequence is the sequence with the highest potential of forming G-quartet. We showed that $13 \%$ of these 954 sites are deleted in the $m d f-1$ such-4; $\operatorname{dog}-1_{F 470}$ genome. This finding raises an important question on the changes in mutation rate when number of mutagenic targets is decreased. Another important question is regarding to the role of $\mathrm{G}_{4}$ DNA in normal development. Recently, $G_{4}$ DNA has been implicated in a variety of biological processes including telomere maintenance, gene expression, epigenetic regulation, and DNA replication [38]. One question to consider is how many $G_{4}$ DNA sites could be removed from a genome yet still maintain viability of the animals.

\section{Methods}

\section{C. elegans strains}

The following mutant alleles were used in this work: unc46(e177), $m d f-1(g k 2), \operatorname{dog}-1(g k 10)$, and such-4(h2168). The following strains were used in this work: KR4233 [unc-46 (e177) mdf-1(gk2) such-4(h2168)] and KR3627 [unc-46 (e177) $m d f-1(g k 2) \quad V / n T 1[l e t-?(m 435)])] ; \quad V C 13$ [dog-1 $(g k 10)]$. Additional strains used in this work were generated in this study. Strains were maintained using standard protocol on nematode growth media (NGM) plates seeded with OP50 bacteria [39]. The strains were maintained at $20^{\circ} \mathrm{C}$. 


\section{Mutation accumulation procedure and phenotypic analysis}

The first suppressor, such-4, was isolated as previously described [24]. Briefly, VC13 was backcrossed to N2 ten times to remove any mutations present in $\mathrm{VC} 13$. Then the outcrossed $d o g-1(g k 10)$ males were used to construct unc-46(e177) mdf-1(gk2) +/+ + nT1[let-X]; dog-1(gk10)/ $\operatorname{dog}-1$ (gk10). Note that unc-46(e177) is linked mdf-1(gk2) and used as a visible marker to track $m d f-1(g k 2)$. $\mathrm{F}_{1} u n c$ $46 m d f-1$ homozygotes $(n=40)$ were picked and plated individually and a single worm, from a plate containing fertile worms in the third generation, was isolated as a suppressor candidate (such-4) [24]. We outcrossed one worm from this strain to establish KR4233 $m d f-1(g k 2)$ such-4(h2168) [6,24], while we maintained a second line at $20^{\circ} \mathrm{C}$ for 470 generations (strain JNC170). Each generation $5 \mathrm{~L}_{4}$ hermaphrodites were transferred to a fresh plate. We also froze the worms at generations 170 (strain JNC168) and 270 (strain JNC169). The phenotypic analysis was performed as previously described [24].

\section{Whole genome sequencing and computational analysis of the unc-46 mdf-1 such-4; dog-1 $1_{\text {F47o }}$ genome}

Genomic DNA was prepared from JNC170 following a standard protocol (http://genetics.wustl.edu/tslab/ protocols/genomic-stuff/worm-genomic-dna-prep/) originally set up by the Andy Fire Lab. The library was prepared with average insert size of $300 \mathrm{bp}$ and the genome was then sequenced using Illumina Solexa sequencing (at Canada's Michael Smith Genome Sciences Centre) and 92,282,948 reads of $101 \mathrm{bp}$ in length were obtained. The reads were then aligned to the $C$. elegans reference genome WS235 (hosted at WormBase) [27] in paired end manner $(46,141,474$ pairs) using the Novoalign alignment tool. $73,482,133(79.63 \%)$ of the total reads were of base quality 30 or more and were uniquely mapped, generating 70-fold coverage of the genome.

SBSs were detected using the uniquely mapped reads and pileup2snp function of the variant caller VarScan2; version 2.3.2 [28]. We used SAMtools [40] to generate the mpileup file necessary as input for VarScan. We filtered out the SBSs that did not meet the following criteria: depth of coverage $>5$ and $\leq 200$, variant frequency $\geq 0.9$ and base quality $\geq 30$. After these filtering steps, we identified 776 substitutions in the $m d f-1$ such-4; $d o g-1_{\mathrm{F} 470}$ genome. However, comparison with the sequenced N2 strains from CGC and Horvitz lab (sequencing reads were kind gift from Dr. Bob Waterston) revealed 525 homozygous substitutions that are unique to mdf-1 such-4; $\operatorname{dog}-1_{\mathrm{F} 470}$ genome. The $251 \mathrm{SBSs}$ were not included in our analysis as they did not accumulate during the course of our experiment. To determine rate of false positives in our set, we randomly selected $30 \mathrm{SBSs}$ (Additional file 2: Table S1A), designed primers flanking the predicted substitution sites, amplified the fragments and re-sequenced using Sanger sequencing method at Genewiz, Inc. All 30 substitutions were confirmed by Sanger re-sequencing, suggesting a false positive rate of less than 5\% (Additional file 2: Table S1A). Also, 12 additional substitutions were confirmed by re-sequencing as a result of being adjacent to randomly selected SBSs or as being nonrandomly selected as candidates in later analyses.

InDels were detected using the uniquely mapped reads and pileup2indel function of the variant caller VarScan2; version 2.3.2 [28]. We used SAMtools [40] to generate the mpileup file necessary as input for VarScan2. We filtered out the InDels that did not meet the following criteria: depth of coverage $>5$ and $\leq 200$, variant frequency $\geq 0.9$ and base quality $\geq 30$. Additionally, we re-evaluated and filtered the output so that each coordinate corresponds to one variant. After these filtering steps, we identified 556 InDels in the mdf-1 such-4; dog-1 $1_{\mathrm{F} 470}$ genome. $\mathrm{r}$, comparison with the sequenced N2 strains from CGC and Horvitz lab (sequencing reads were kind gift from Dr. Bob Waterston) revealed 150 homozygous InDels that are unique to mdf-1 such-4; $\operatorname{dog}-1_{\mathrm{F} 470}$ genome. The $406 \mathrm{InDels}$ were not included in our analysis as they did not accumulate during the course of our experiment. To determine rate of false positives in our set, we have randomly selected 25 InDels (Additional file 2: Table S3A), designed primers flanking the predicted InDel sites, amplified the fragments and re-sequenced using Sanger sequencing method at Genewiz, Inc. All of the 25 InDels were confirmed by Sanger re-sequencing, suggesting a false positive rate of less than 5\% (Additional file 2: Table S3A).

These unique-mapping reads were then used, together with Pindel [33], to identify deletions. The final set of fixed/homozygous deletions was selected based on the following criteria: $a=$ the number of unique reads supporting the breakpoints of the deletion; $b=$ the number of reads within the deleted region; select the predicted deletion if $\frac{a}{a+b}$ is larger than 0.5 and the size of the deletion is larger than 20 bp. From the candidate deletions, 28 were randomly selected and the regions were PCRamplified using the same genomic DNA that was used for the WGS and primers designed in the flanking regions of the computationally identified deletions. All of the 28 randomly selected predicted deletion sizes were confirmed using DNA electrophoresis gels, suggesting the rate of false positives of less than $5 \%$.

\section{Whole genome sequencing and computational analysis of the unc-46 mdf-1 such-4; dog-1 $1_{\text {F170 }}$ and mdf-1 such-4; dog-1 $1_{\text {F27o }}$ strains}

The genomic DNA was prepared from JNC168 and JNC169 following a standard protocol (http://genetics.wustl.edu/ tslab/protocols/genomic-stuff/worm-genomic-dna-prep/). 
The genomic DNA was sheared to generate $500 \mathrm{bp}$ fragment and the library was prepared using the NEBNext ${ }^{\circ}$ Ultra $^{\text {Tu }}$ DNA Library Prep Kit for Illumina ${ }^{\circ}$. The library was then sequenced using Illumina Solexa sequencing (at Simon Fraser University) and 9,950,748 $\left(\mathrm{F}_{170}\right)$ and $6,225,488\left(\mathrm{~F}_{270}\right)$ reads of $250 \mathrm{bp}$ length were obtained. The reads were then aligned to the $C$. elegans reference genome WS235 (hosted at WormBase) [27] in paired end manner using the Novoalign alignment tool as described above to achieve 20 -fold $\left(\mathrm{F}_{170}\right)$ and 14-fold $\left(\mathrm{F}_{270}\right)$ coverage. For both strains, we used Pindel [33] to identify deletions and duplications, and VarScan2; version 2.3.2 [28] to identify small InDels and substitutions, as described above. We also used sequenced N2 strains from CGC and Horvitz lab to remove variants existing in the N2 strain. Then, we compared the variants present in the $\mathrm{F}_{170}$ and $\mathrm{F}_{270}$ genomes with the ones identified in the $\mathrm{F}_{470}$ genome.

\section{oaCGH analysis of the unc-46 mdf-1 such-4; dog-1 strains}

To perform oaCGH analysis, we used the same genomic DNA from the mdf-1 such-4; dog-1 lines $\left(\mathrm{F}_{170}, \mathrm{~F}_{270}\right.$ and $\mathrm{F}_{470}$ ) that were used for the WGS and the reference N2 DNA that was prepared following a standard protocol. oaCGH analysis was performed as described by Maydan and colleagues [41] with a newly designed microarray. The 3-plex microarray with design name 120618_Cele_WS230_JK_CGH was manufactured by Roche NimbleGen Inc. with each individual sub-array comprising $720 \mathrm{k} 50$-mer oligonucleotide probes. The filters used to select the probes primarily followed Maydan and colleagues [41] without focusing on coding regions in order to provide a more uniform coverage of the genome (WormBase release WS230). In regions where unique probes could not be designed selection filters were slightly relaxed in order to allow the inclusion of probes with possible cross-hybridization to at most one other location in the genome.

\section{Availability of supporting data}

Whole Genome Sequencing (WGS) data, fastq files, for the three strains: JNC168, JNC169 and JNC170 are available in the NCBI Sequence Read Archive (SRA) (http://www.ncbi. nlm.nih.gov/sra) under the BioProject accession number SRP053517 (PRJNA275156) with the fastq files: (170_R1. fastq, 170_R2.fastq, 270_R1.fastq, 270_R2.fastq, 470_R1. fastq and 470_R2.fastq) under the SRR1797354 accession number.

\section{Additional files}

Additional file 1: Figure S1. Mutation accumulation in unc-46 mdf-1 such-4; dog-1 strains. Accumulation of mutations was visualized using Circos [42]. Note that due to the limited resolution, data points that occur close in the genome may appear as a single line or a single link.
$F_{170}$ chromosomes and variants originating at $F_{170}$ are depicted in green; $F_{270}$ chromosomes and variants originating at $F_{270}$ are depicted in purple, while $F_{470}$ chromosomes and variants unique to $F_{470}$ are depicted in blue. The outer circle is a plot of all the variants present at a specific generation, while inner links are depicting propagation of the variants from one generation time-point to the next. The following variants are shown: (A) SBSs, (B) InDels, (C) Duplications, (D) Deletions.

Additional file 2: Table S1A. Homozygous SBSs identified in the $\mathrm{F}_{170}$ : $F_{270}$ and $F_{470}$ genomes. Table S1B. Homozygous SBSs identified after propagating $m d f-1$; dog-1; such-4 for 300 generations. Table S2. Location of the $\mathrm{G}_{3-5} \mathrm{~N}_{1-7} \mathrm{G}_{3-5} \mathrm{~N}_{1-7} \mathrm{G}_{3-5} \mathrm{~N}_{1-7} \mathrm{G}_{3-5}$ sequences in the $\mathrm{C}$. elegans genome. Table S3A. Homozygous InDels smaller than or equal to $20 \mathrm{bp}$ identified in the $F_{170} ; F_{270}$ and $F_{470}$ genomes. Table S3B. Homozygous InDels smaller than or equal to $20 \mathrm{bp}$ after propagating $m d f-1$; dog-1; such-4 for 300 generations. Table S4A. Duplications identified in the $F_{170} ; F_{270}$ and $\mathrm{F}_{470}$ genomes. Table S4B. Duplications identified after propagating mdf-1; dog-1; such-4 for 300 generations. Table S5A. Homozygous deletions larger than $20 \mathrm{bp}$ identified in the $F_{170 ;} F_{270}$ and $F_{470}$ genomes. Table S5B. Homozygous deletions larger than 20 bp identified after propagating $m d f-1$; $\operatorname{dog}_{-1}$; such-4 for 300 generations. Table S6. Accumulation of mutations predicted to disrupt protein-coding genes after 300 generations of propagation. Table S7. All mutations predicted to disrupt protein-coding genes in the $m d f-1$; dog-1; such-4 $\mathrm{F}_{470}$ genome.

\section{Abbreviations}

SAC: Spindle assembly checkpoint; APC/C: Anaphase promoting complex or cyclosome; CIN: Chromosome instability; WGS: Whole genome sequencing; FA: Fanconi anemia; oaCGH: oligonucleotide array Comparative Genomic Hybridization; G4: G-quartet; SBS: Single base substitution; GV: Genome variant.

\section{Competing interests}

The authors declare that they have no competing interests.

\section{Authors' contributions}

MTG made the strains; MTG and TW wrote Perl scripts; MTG and ZQ prepared DNA for WGS and oaCGH. SF and JT generated oaCGH data. MTG analyzed the WGS data. MTG and SF analyzed the oaCGH data. MTG, DGM, AMR and NC led the study. MTG wrote the manuscript. All authors read, edited and approved the final manuscript.

\section{Acknowledgements}

We thank the C. elegans Gene Knockout Consortium for generating the deletion mutants, the Caenorhabditis Genetics Center (CGC) for providing the strains, and the Michael Smith Genome Sciences Centre for Illumina sequencing. We also thank Bob Waterston for the sequencing reads of N2 strains; Robert Johnsen, Steven Jones, Harald Hutter and Nancy Hawkins for comments. This work was supported by the Canadian Institutes for Health Research (CIHR) and Fanconi Anemia Fellowship to MTG, and the Discovery Grant from the Natural Science and Engineering Research Council (NSERC) to NC. NC is a Michael Smith Foundation for Health Research (MSFHR) Scholar and a CIHR New Investigator. Work in the laboratory of DGM is supported by a grant from CIHR. DGM is a Senior Fellow of the Canadian Institute for Advanced Research (CIFAR). Work in the laboratory of AMR is supported by grants from CIHR and NSERC.

\section{Author details}

'Department of Molecular Biology and Biochemistry, Simon Fraser University, V5A 156 Burnaby, BC, Canada. ²Department of Zoology, University of British Columbia, V6T 1 Z4 Vancouver, BC, Canada. ${ }^{3}$ Department of Medical Genetics, University of British Columbia, V6T 1 Z3 Vancouver, BC, Canada. ${ }^{4}$ Current affiliation: Centre for Molecular Medicine and Therapeutics; Child and Family Research Institute, Vancouver, BC, Canada. ${ }^{5}$ Current affiliation: Treatable Intellectual Disability Endeavour in British Columbia, Vancouver, BC, Canada.

Received: 3 September 2014 Accepted: 24 February 2015

Published online: 18 March 2015 


\section{References}

1. Burrell RA, McGranahan N, Bartek J, Swanton C. The causes and consequences of genetic heterogeneity in cancer evolution. Nature. 2013:501:338-45.

2. Kitagawa R, Rose AM. Components of the spindle-assembly checkpoint are essential in Caenorhabditis elegans. Nat Cell Biol. 1999:1:514-21.

3. Musacchio A, Salmon ED. The spindle-assembly checkpoint in space and time. Nat Rev Mol Cell Biol. 2007:8:379-93.

4. Cheung I, Schertzer M, Rose A, Lansdorp PM. Disruption of dog-1 in Caenorhabditis elegans triggers deletions upstream of guanine-rich DNA Nat Genet. 2002;31:405-9.

5. Kruisselbrink E, Guryev V, Brouwer K, Pontier DB, Cuppen E, Tijsterman M Mutagenic capacity of endogenous G4 DNA underlies genome instability in FANCJ-defective C. elegans. Curr Biol. 2008;18:900-5.

6. Zhao Y, Tarailo-Graovac M, O'Neil NJ, Rose AM. Spectrum of mutational events in the absence of DOG-1/FANCJ in Caenorhabditis elegans. DNA Repair. 2008;7:1846-54

7. Sen D, Gilbert W. Formation of parallel four-stranded complexes by guanine-rich motifs in DNA and its implications for meiosis. Nature. 1988:334:364-6.

8. Sen D, Gilbert W. A sodium-potassium switch in the formation of fourstranded G4-DNA. Nature. 1990;344:410-4.

9. Gellert M, Lipsett MN, Davies DR. Helix formation by guanylic acid. Proc Natl Acad Sci U S A. 1962;48:2013-8.

10. Youds JL, Barber LJ, Ward JD, Collis SJ, O'Neil NJ, Boulton SJ, et al. DOG-1 is the Caenorhabditis elegans BRIP1/FANCJ homologue and functions in interstrand cross-link repair. Mol Cell Biol. 2008:28:1470-9.

11. Huppert $J$, Balasubramanian S. Prevalence of quadruplexes in the human genome. Nucleic Acids Res. 2005:33:2908-16.

12. Todd AK, Johnston M, Neidle S. Highly prevalent putative quadruplex sequence motifs in human DNA. Nucleic Acids Res. 2005:33:2901-7.

13. Levitus M, Waisfisz Q, Godthelp BC, de Vries Y, Hussain S, Wiegant WW, et al. The DNA helicase BRIP1 is defective in Fanconi anemia complementation group. J Nat Genet. 2005;37:934-5.

14. Levran O, Attwooll C, Henry RT, Milton KL, Neveling K, Rio P, et al. The BRCA1-interacting helicase BRIP1 is deficient in Fanconi anemia. Nat Genet. 2005;37:931-3

15. Litman $\mathrm{R}$, Peng $\mathrm{M}$, Jin $Z$, Zhang $F$, Zhang J, Powell $\mathrm{S}$, et al. BACH1 is critical for homologous recombination and appears to be the Fanconi anemia gene product FANCJ. Cancer Cell. 2005:8:255-65.

16. Seal S, Thompson D, Renwick A, Elliott A, Kelly P, Barfoot R, et al. Truncating mutations in the Fanconi anemia J gene BRIP1 are low-penetrance breast cancer susceptibility alleles. Nat Genet. 2006;38:1239-41.

17. Cantor SB, Bell DW, Ganesan S, Kass EM, Drapkin R, Grossman S, et al. $\mathrm{BACH1}$, a novel helicase-like protein, interacts directly with BRCA1 and contributes to its DNA repair function. Cell. 2001;105:149-60.

18. Youds JL, O'Neil NJ, Rose AM. Homologous recombination is required for genome stability in the absence of DOG-1 in Caenorhabditis elegans. Genetics. 2006;173:697-708.

19. Pontier DB, Kruisselbrink E, Guryev V, Tijsterman M. Isolation of deletion alleles by G4 DNA-induced mutagenesis. Nat Methods. 2009:6:655-7.

20. Lynch M, Sung W, Morris K, Coffey N, Landry CR, Dopman EB, et al. A genome-wide view of the spectrum of spontaneous mutations in yeast. Proc Natl Acad Sci U S A. 2008;105:9272-7.

21. Denver DR, Dolan PC, Wilhelm $\amalg$, Sung W, Lucas-Lledó J, Howe DK, et al. A genome-wide view of Caenorhabditis elegans base-substitution mutation processes. Proc Natl Acad Sci U S A. 2009:106:16310-4.

22. Ossowski S, Schneeberger K, Lucas-Lledó Jl, Warthmann N, Clark RM, Shaw $\mathrm{RG}$, et al. The rate and molecular spectrum of spontaneous mutations in Arabidopsis thaliana. Science. 2010;327:92-4.

23. Keightley PD, Trivedi U, Thomson M, Oliver F, Kumar S, Blaxter ML. Analysis of the genome sequences of three Drosophila melanogaster spontaneous mutation accumulation lines. Genome Res. 2009:19:1195-201.

24. Tarailo M, Kitagawa R, Rose AM. Suppressors of spindle checkpoint defect (such) mutants identify new mdf-1/MAD1 interactors in Caenorhabditis elegans. Genetics. 2007:175:1665-79.

25. Tarailo-Graovac M, Wang J, Tu D, Baillie DL, Rose AM, Chen N. Duplication of cyb-3 (cyclin B3) suppresses sterility in the absence of mdf-1/MAD1 spindle assembly checkpoint component in Caenorhabditis elegans. Cell Cycle Georget Tex. 2010;9:4858-65.
26. Tarailo-Graovac M, Wong T, Qin Z, Flibotte S, Tylor J, Moerman DG, et al. Cyclin B3 and dynein heavy chain cooperate to increase fitness in the absence of mdf-1/MAD1 in Caenorhabditis elegans. Cell Cycle. 2014;13:1-11.

27. Yook K, Harris TW, Bieri T, Cabunoc A, Chan J, Chen WJ, et al. WormBase 2012: more genomes, more data, new website. Nucleic Acids Res. 2012;40 (Database issue):D735-41.

28. Koboldt DC, Zhang Q, Larson DE, Shen D, McLellan MD, Lin L, et al. VarScan 2: somatic mutation and copy number alteration discovery in cancer by exome sequencing. Genome Res. 2012;22:568-76.

29. Denver DR, Morris K, Lynch M, Thomas WK. High mutation rate and predominance of insertions in the Caenorhabditis elegans nuclear genome. Nature. 2004;430:679-82

30. Zhu YO, Siegal ML, Hall DW, Petrov DA. Precise estimates of mutation rate and spectrum in yeast. Proc Natl Acad Sci U S A. 2014;111:E2310-8.

31. Chen J-Q, Wu Y, Yang H, Bergelson J, Kreitman M, Tian D. Variation in the ratio of nucleotide substitution and indel rates across genomes in mammals and bacteria. Mol Biol Evol. 2009:26:1523-31.

32. Lipinski KJ, Farslow JC, Fitzpatrick KA, Lynch M, Katju V, Bergthorsson U High spontaneous rate of gene duplication in Caenorhabditis elegans. Curr Biol. 2011;21:306-10

33. Ye K, Schulz MH, Long Q, Apweiler R, Ning Z. Pindel: a pattern growth approach to detect break points of large deletions and medium sized insertions from paired-end short reads. Bioinformatics. 2009;25:2865-71.

34. Kallioniemi A, Kallioniemi OP, Sudar D, Rutovitz D, Gray JW, Waldman F, et al. Comparative genomic hybridization for molecular cytogenetic analysis of solid tumors. Science. 1992:258:818-21.

35. Verma A, Yadav VK, Basundra R, Kumar A, Chowdhury S. Evidence of genome-wide G4 DNA-mediated gene expression in human cancer cells. Nucleic Acids Res. 2009;37:4194-204.

36. Vergara IA, Frech $C$, Chen N. Coovar: co-occurring variant analyzer. BMC Res Notes. 2012;5:615

37. Zhao Y, O'Neil NJ, Rose AM. Poly-G/poly-C tracts in the genomes of Caenorhabditis. BMC Genomics. 2007:8:403.

38. Tarsounas M, Tijsterman M. Genomes and G-quadruplexes: for better or for worse. J Mol Biol. 2013:425:4782-9.

39. Brenner $S$. The genetics of Caenorhabditis elegans. Genetics. 1974;77:71-94

40. Li H, Handsaker B, Wysoker A, Fennell T, Ruan J, Homer N, et al. The Sequence Alignment/Map format and SAMtools. Bioinformatics. 2009;25:2078-9.

41. Maydan JS, Flibotte S, Edgley ML, Lau J, Selzer RR, Richmond TA, et al. Efficient high-resolution deletion discovery in Caenorhabditis elegans by array comparative genomic hybridization. Genome Res. 2007;17:337-47.

42. Krzywinski M, Schein J, Birol I, Connors J, Gascoyne R, Horsman D, et al. Circos: an information aesthetic for comparative genomics. Genome Res. 2009;19:1639-45.

\section{Submit your next manuscript to BioMed Central and take full advantage of:}

- Convenient online submission

- Thorough peer review

- No space constraints or color figure charges

- Immediate publication on acceptance

- Inclusion in PubMed, CAS, Scopus and Google Scholar

- Research which is freely available for redistribution 